VIDEO

\title{
A comprehensive examination of the novel techniques used for in vivo and ex vivo confocal laser endomicroscopy of pancreatic cystic lesions
}
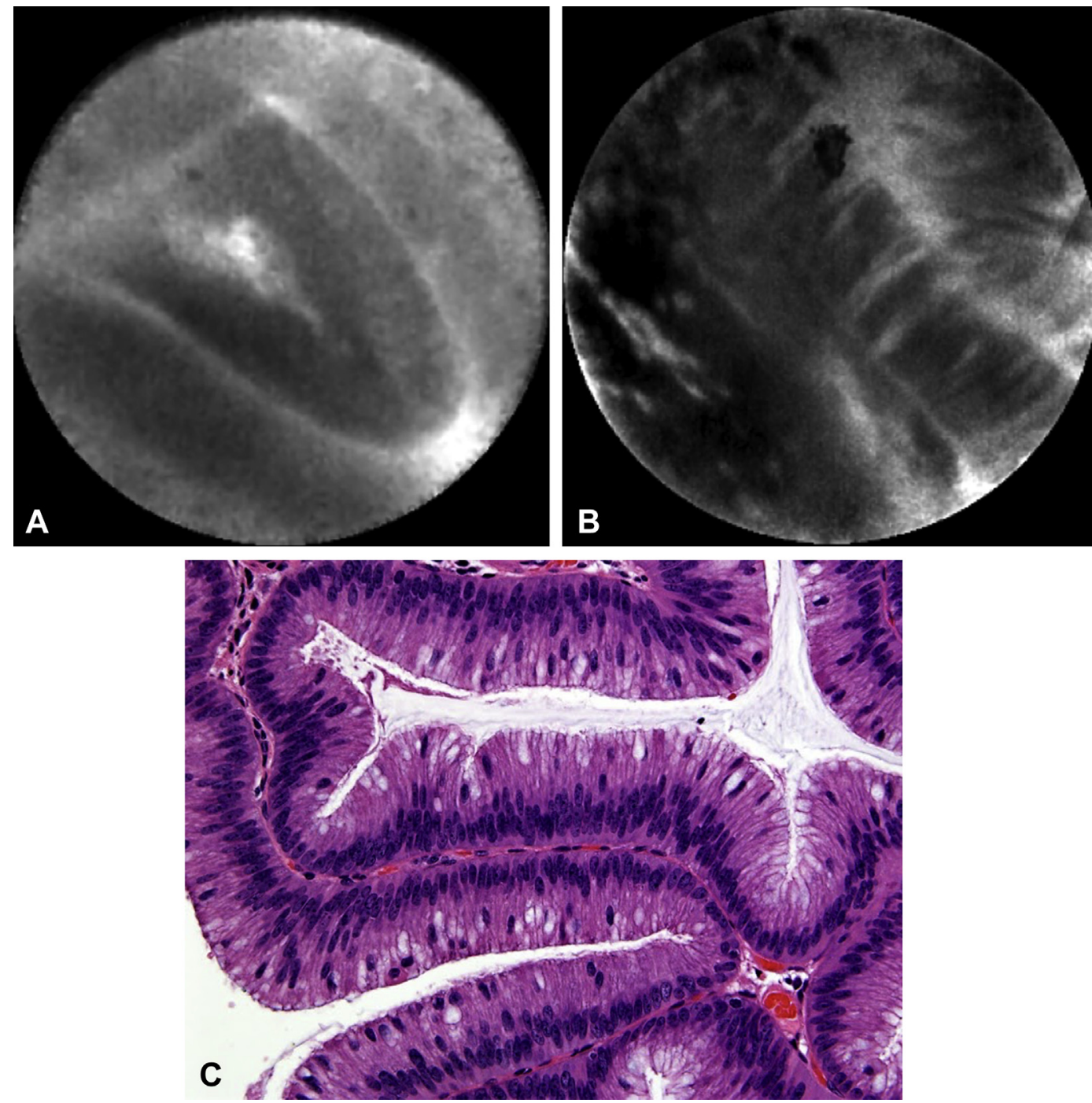

Figure 1. A, In vivo needle-based confocal laser endomicroscopic view of an intraductal papillary mucinous neoplasm. B, Ex vivo confocal laser endomicroscopic view of an intraductal papillary mucinous neoplasm. Both demonstrate fingerlike projections, or papillae. C, Histopathologic view of an intraductal papillary mucinous neoplasm showing tall columnar epithelium with elongated nuclei and goblet cells (H\&E, orig. mag. $\times 40$ ).

Confocal laser endomicroscopy (CLE) is a new technique for the evaluation of pancreatic cystic lesions (PCLs). To our knowledge, there are no studies validating in vivo EUS-guided needle-based CLE (nCLE) images by ex vivo CLE and surgical histopathologic examination. This video presents the technique of in vivo and ex vivo CLE for the evaluation of PCLs. For in vivo imaging, an AQ-Flex nCLE miniprobe (Cellvizio, Mauna Kea 
Technologies, Paris, France) was preloaded into a 19-gauge Flex needle (Boston Scientific, Natick, Mass). The technique is demonstrated in Video 1 . For ex vivo imaging, in the operating room, $5 \mathrm{~mL} \mathrm{10 \%} \mathrm{fluorescein}$ was intravenously injected before ligation of blood vessels supplying the pancreas. After resection, an 18-gauge hypodermic needle was used to puncture the cyst, and the nCLE miniprobe was introduced into the cyst to capture images of the entire epithelium. Subsequently, the PCL was opened, site-specific epithelial biopsy specimens were obtained, and correlated highresolution ex vivo images were recorded with a Gastroflex ultra-high-definition probe (Cellvizio; Mauna Kea Technologies, Paris, France). The ex vivo and histopathologic findings validated the in vivo nCLE findings, as demonstrated in Figure 1. To our knowledge, this is the first study to demonstrate the in vivo and ex vivo CLE techniques for PCLs.

\section{DISCLOSURE}

All authors disclosed no financial relationships relevant to this publication.

Amrit K. Kamboj, MD, The Ohio State University College of Medicine, Rohan M. Modi, MD, Department of Internal Medicine, Benjamin Swanson, MD, PhD, Department of Pathology, Darwin L. Conwell, MD, MS, Somashekar G. Krishna, MD, MPH, Division of Gastroenterology, Hepatology, and Nutrition, The Ohio State University Wexner Medical Center, Columbus, Ohio, USA

Copyright (c) 2016 The Authors. Published by Elsevier, Inc. on behalf of the American Society for Gastrointestinal Endoscopy. This is an open access article under the CC BY-NC-ND license (http://creativecommons.org/ licenses/by-nc-nd/4.0/).

http://dx.doi.org/10.1016/j.vgie.2016.07.002 